\title{
Increased levels of ligands of Toll-like receptors 2 and 4 in type 1 diabetes
}

\author{
S. Devaraj • M. R. Dasu • S. H. Park • I. Jialal
}

Received: 17 March 2009/Accepted: 24 April 2009/Published online: 20 May 2009

(C) The Author(s) 2009. This article is published with open access at Springerlink.com

\begin{abstract}
Aims/hypothesis Type 1 diabetes is a proinflammatory state characterised by increased levels of circulating biomarkers of inflammation and monocyte activity. We have shown increased Toll-like receptor 2 (TLR2) and TLR4 expression and signalling in monocytes from type 1 diabetic patients. Several endogenous ligands of TLR2 and TLR4 have been identified; however, there is a paucity of data on levels of these endogenous ligands in diabetes. Thus, the aim of this study was to examine circulating levels of exogenous/ endogenous ligands of TLR2 and TLR4 in type 1 diabetic patients and to compare these with the levels in matched healthy controls.

Methods Healthy controls $(n=37)$ and type 1 diabetic patients $(n=34)$ were recruited, and a fasting blood sample was obtained. Circulating levels of endotoxin, heat-shock protein 60 (Hsp60), high-mobility group box 1 (HMGB1) and growth arrest-specific 6 (GAS6) proteins were assessed by ELISA, and TLR2 and TLR4 expression was determined by flow cytometry.

Results Levels of the classical TLR4 ligand, endotoxin, were significantly elevated in type 1 diabetic patients compared with those in matched controls. Hsp60 and HMGB1 concentrations were also significantly increased
\end{abstract}

S. Devaraj $\cdot$ M. R. Dasu $\cdot$ S. H. Park $\cdot$ I. Jialal $(\bowtie)$

Laboratory for Atherosclerosis and Metabolic Research,

UC Davis Medical Center,

4635 II Ave, Res. 1 Bldg, Rm 3000,

Sacramento, CA 95817, USA

e-mail: ishwarlal.jialal@ucdmc.ucdavis.edu

S. Devaraj · I. Jialal

VA Medical Center,

Mather, CA, USA in the patients $(p<0.01$ and $p<0.001$, respectively). No significant differences were observed in GAS6.

Conclusions/interpretation We report the novel observation that levels of ligands of TLR2 and TLR4 are significantly elevated in type 1 diabetes, and this, in concert with hyperglycaemia, accounts for the increase in TLR2 and TLR4 activity, underscoring the proinflammatory state of type 1 diabetes.

Keywords Endotoxin · HMGB1 - Hsp60 - Inflammation · Ligand $\cdot$ Toll-like receptor
Abbreviations
GAS6 Growth arrest-specific 6
HMGB1 High-mobility group box 1
hsp Heat shock protein
IFN $\beta \quad$ Interferon- $\beta$
mfi Mean fluorescence intensity of 10,000 cells
MyD88 Myeloid differentiation primary response 88
NF- $k B$ Nuclear factor $k B$
RAGE Receptor for advanced glycation end-products
TLR Toll-like receptor

\section{Introduction}

Type 1 diabetes is associated with an increased risk of micro- and macrovascular complications [1]. Recent studies have shown that type 1 diabetes is a proinflammatory state [2]. Members of the Toll-like receptor (TLR) family play a critical role in innate immunity [3]. The TLRs, TLR2 and TLR4 also play an important role in atherosclerosis [3]. We recently demonstrated increased levels of TLR2 and TLR4 expression, activity and signalling in monocytes of type 1 
diabetic patients compared with those in matched controls [4].

A number of endogenous molecules may also be potent activators of the innate immune system [5] and are capable of inducing the release of proinflammatory cytokines from monocytes/macrophages via the TLR signal-transduction pathways. However, there are limited data on levels of endogenous ligands of TLR 2 and TLR4 in type 1 diabetes. The major ligand involved in TLR4 activation is endotoxin [5]. The activation of these TLR2 and TLR4 on monocytes leads to the production of cytokines and chemokines. Heat shock proteins (hsps) are endogenous ligands of TLR2 and TLR4 [6]. Using macrophages from $\mathrm{C} 3 \mathrm{H} / \mathrm{HeJ}$ mice with a Tlr4 point mutation, the cytokine effects of recombinant human Hsp60 were shown to be dependent on TLR4, suggesting that Hsp60 may be a TLR4 ligand [6]. Highmobility group box 1 (HMGB1) protein has been reported to be a 'late' proinflammatory mediator in sepsis. HMGB1 can induce activation of intracellular signalling pathways via activation of TLR2, TLR4 and the receptor for advanced glycation end-products (RAGE) [7]. Extracellular HMGB1 thus acts as an alarmin and can activate the innate system and function in a synergistic fashion with other proinflammatory mediators to induce responses. Growth arrest-specific 6 (GAS6) protein is another endogenous ligand of TLR2 and TLR4 that is induced in experimental models of diabetic nephropathy [8]. Thus, in this study, since type 1 diabetes has previously been shown to be a proinflammatory state involving increased TLR2 and TLR4 expression, we determined the levels of endogenous (Hsp60, HMGB1 and GAS6) and exogenous (endotoxin) ligands of TLR2 and TLR4 in patients with type 1 diabetes and compared these with the levels in matched healthy controls. In a previous report [4], we examined myeloid differentiation response 88 (MyD88)-dependent TLR activation, and we follow up this work in the present study by examining levels of interferon- $\beta$ (IFN $\beta$ ), a biological indicator of non-MyD88-dependent activation of TLRs.

\section{Methods}

Type 1 diabetic patients $(n=34)$ (aged $<20$ years at onset and on insulin therapy since diagnosis) with a duration of diabetes of $\geq 1$ year were recruited from the diabetes/ pediatric clinics at UC Davis Medical Center and advertisements. All patients were aged $\geq 18$ years at recruitment and none was receiving metformin and/or thiazolidinediones, or any anti-inflammatory therapy.

Healthy controls $(n=37)$ aged $>18$ years were included if they had a normal complete blood count, no family history of diabetes or other chronic diseases, normal kidney, liver, thyroid function and fasting plasma glucose $<5.5 \mathrm{mmol} / \mathrm{l}$.
Selection criteria were as described previously [4]. Informed consent was obtained from participants in the study, which was approved by the Institutional Review Board at UC Davis Medical Center. For each participant, a medical history was obtained, a physical examination was undertaken, and then a fasting blood sample $(30 \mathrm{ml})$ was collected. Routine laboratory tests were performed in the clinical pathology laboratory.

Mononuclear cells were isolated from the heparinised blood samples by Ficoll-Hypaque centrifugation followed by negative magnetic separation using the depletion technique (Miltenyi Biotech, Auburn, CA, USA) [4]. The purity of the isolated mononuclear cells was $88 \%$. Monocytes from control and type 1 diabetic patients were incubated with anti-human TLR2 and TLR4 antibodies (Invivogen, San Diego, CA, USA) or isotype controls, and surface expression of TLR2 and TLR4 was analysed using BD FACSArray (Becton-Dickinson, Fullerton, CA, USA) [4]. Results were expressed as mean fluorescence intensity of 10,000 cells (mfi). The intra- and inter-assay CVs for TLR2 and TLR4 expression were both found to be $<5 \%$ and $<15 \%$, respectively. Endotoxin levels were measured in serum samples of controls and type 1 diabetic patients by the Limulus amoebocyte lysate assay (Invivogen). Sandwich ELISA was used to assess levels of IFN $\beta$ (R\&D, Minneapolis, MN, USA), HMGB1 (Shino Test, Tokyo, Japan), Hsp60 (Assay Designs, Ann Arbor, MI, USA) and GAS6 (R\&D, Minneapolis, MN, USA); the intra-assay CV for each of these assays was $<10 \%$.

Statistical analyses were performed using SAS (SAS Institute, Cary, NC, USA). Data are expressed as the mean \pm SD for parametric data and as the median and interquartile range for non-parametric data. Parametric data were analysed using paired, two-tailed $t$ tests; non-parametric data were analysed using Wilcoxon signed rank tests. A $p$ value of less than 0.05 was considered significant. Spearman's rank correlation was computed to assess associations between variables.

\section{Results}

There were no significant differences between the control and type 1 diabetic groups in terms of age, BMI, male/ female ratio and lipid profile. Levels of glucose and $\mathrm{HbA}_{1 \mathrm{c}}$ were significantly higher in type 1 diabetic patients than in controls (Table 1).

Levels of the major exogenous ligand for TLR4, endotoxin, were significantly increased in type 1 diabetic patients compared with those in the controls (Table 2). Levels of Hsp60 and HMGB1 were also significantly elevated $(p<0.01$ and $p<0.001$, respectively). There was no significant difference between the two groups with 
Table 1 Characteristics of the participants at baseline

Data are expressed as mean $\pm \mathrm{SD}$

${ }^{*} p<0.05$ vs controls

\begin{tabular}{lll}
\hline & Controls $(n=37)$ & Type 1 diabetes $(n=34)$ \\
\hline Age $($ years $)$ & $34 \pm 11$ & $32 \pm 11$ \\
BMI $\left(\mathrm{kg} / \mathrm{m}^{2}\right)$ & $24 \pm 4$ & $25 \pm 4$ \\
Male/female ratio & $19: 18$ & $15: 19$ \\
Glucose $(\mathrm{mmol} / \mathrm{l})$ & $4.9 \pm 0.5$ & $7.2 \pm 3.8^{*}$ \\
HbA $_{1 \mathrm{c}}(\%)$ & $5.4 \pm 0.4$ & $7.9 \pm 1.4^{*}$ \\
Total cholesterol $(\mathrm{mmol} / \mathrm{l})$ & $4.5 \pm 0.8$ & $4.7 \pm 0.8$ \\
Triacylglycerol $(\mathrm{mmol} / \mathrm{l})$ & $0.9 \pm 0.4$ & $0.9 \pm 0.5$ \\
LDL-cholesterol $(\mathrm{mmol} / \mathrm{l})$ & $2.9 \pm 0.6$ & $2.9 \pm 0.6$ \\
HDL-cholesterol $(\mathrm{mmol} / \mathrm{l})$ & $1.1 \pm 0.4$ & $1.3 \pm 0.4$ \\
\hline
\end{tabular}

respect to GAS6 levels. IFN $\beta$ concentrations were significantly higher in type 1 diabetic patients compared with controls $(43.9 \pm 10.3$ vs $26.7 \pm 3.2 \mathrm{nmol} / \mathrm{l}$, respectively, $p<0.05$ ).

Across the study sample as a whole, there was a significant correlation between TLR2 and HMGB1 levels $(r=0.42, p<0.05)$, a trend towards a positive correlation between TLR2 and Hsp60 levels (though this did not reach statistical significance; $r=0.33, p<0.09)$, and no correlation between TLR2 and endotoxin $(r=0.34, p>0.2)$. TLR4 was significantly correlated with endotoxin $(r=0.56, p<0.01)$ and Hsp60 ( $r=0.61, p<0.01)$, and showed a trend towards a positive correlation with HMGB1 (though this did not reach statistical significance; $r=0.39, p<0.1$ ). The only ligand to be significantly correlated with $\mathrm{HbA}_{1 \mathrm{c}}$ was endotoxin $(r=0.64, p<0.01)$.

\section{Discussion}

In addition to regulating inflammation, both TLR2 and TLR4 are relevant to atherosclerosis [3]. The expression and activity of TLR2 and TLR4 are increased in monocytes from type 1 diabetic patients [4]. Downstream targets of TLR, nuclear factor-kB (NF-kB), MyD88, TIR-domain-containing adapter-inducing IFN- $\beta$ (TRIF) and phosphorylated IL-1 receptor-associated kinase (pIRAK), were significantly upre-

Table 2 Levels of ligands of TLR2 and TLR4

\begin{tabular}{lcc}
\hline & Controls $(n=37)$ & Type 1 diabetes $(n=34)$ \\
\hline Endotoxin $(\mathrm{nmol} / \mathrm{l})$ & $2.53 \pm 0.67$ & $3.32 \pm 0.82^{* *}$ \\
HMGB1 $(\mathrm{nmol} / \mathrm{l})$ & $0.13 \pm 0.04$ & $0.20 \pm 0.06^{* *}$ \\
Hsp60 $(\mathrm{pmol} / \mathrm{l})$ & $18.5 \pm 5.0$ & $23.2 \pm 6.7^{* *}$ \\
GAS6 $(\mathrm{nmol} / \mathrm{l})$ & $11.2 \pm 6.9$ & $10.2 \pm 5.9$ \\
\hline
\end{tabular}

Data are expressed as mean $\pm \mathrm{SD}$

${ }^{* *} p<0.01$ vs controls gulated in type 1 diabetes [4]. The release of IL-1 $\beta$ and TNF- $\alpha$ from monocytes was significantly increased in type 1 diabetic patients compared with controls and was correlated with TLR2 and TLR4 expression [4]. Also, high glucose levels upregulate, and insulin downregulates, TLR2 and TLR4 expression [9].

Given the importance of TLR2 and TLR4 in atherosclerosis and their emerging role in the proinflammatory state of type 1 diabetes, in this study we examined circulating levels of several different ligands of TLR2 and TLR4 in patients with type 1 diabetes and compared these with the levels in matched controls. Creely et al. [10] have previously shown increased levels of endotoxin in type 2 diabetes but not increased TLR4 expression in adipose tissue. Furthermore, they demonstrated that circulating endotoxin levels are associated with atherosclerosis [10]. In this report, we provide evidence of increased endotoxin levels, which could contribute to the proinflammatory burden by activation of TLR4 and downstream signalling pathways.

The hsps such as Hsp60 induce the production of proinflammatory cytokines via activation of TLR2 and TLR4 [6]. In this paper we show that type 1 diabetic patients also have high circulating levels of Hsp60, which could trigger the activation of TLR2 and TLR4, leading to a proinflammatory state.

HMGB1 induces the activation of intracellular signalling pathways via interaction with at least three patternrecognition receptors: TLR2, TLR4 and RAGE. Furthermore, HMGB1 stimulates the transcription of genes encoding proteins involved in the proinflammatory state [7]. In the present study, we show that type 1 diabetic patients have high circulating levels of HMGB1, which could trigger TLR2 and TLR4 activation, leading to a proinflammatory state.

TLRs mainly signal through the adapter protein MyD88 via activation of NF- $\mathrm{KB}$ and mitogen-activated protein kinase, resulting in the increased transcription of inflammationrelated genes, such as those encoding IL-1, TNF- $\alpha$, MCP-1 [3]. In addition, a MyD88-independent pathway involving 
Trif is essential to TLR3 and TLR4 signalling and induces IFN $\beta$ [3]. In the previous study [4], we showed that activation of the MyD88 pathway is increased in type 1 diabetic patients. In the present study, we show that type 1 diabetic patients have high circulating levels of IFN $\beta$ (downstream indicator of non-MyD88 pathway activation).

In summary, in this paper we report the novel observation that there is significant elevation of endogenous ligands of TLR2 and TLR4 in type 1 diabetes, which, in concert with hyperglycaemia, contributes to the increase in TLR2 and TLR4 signalling that results in the proinflammatory state of type 1 diabetes. In future studies, we will test levels of endogenous ligands and TLR2 and TLR4 expression and signalling in type 2 diabetes, which is also a proinflammatory state.

Acknowledgements Grant support from the Juvenile Diabetes Research Foundation (JDRF) (no. 1-2007-585 to I. Jialal) and the National Institutes of Health (no. DK69801 to S. Devaraj; no. K24AT00596 to I. Jialal). Technical support from C. Duncan-Staley.

Duality of interest The authors declare that there is no duality of interest associated with this manuscript.

Open Access This article is distributed under the terms of the Creative Commons Attribution Noncommercial License which permits any noncommercial use, distribution, and reproduction in any medium, provided the original author(s) and source are credited.

\section{References}

1. Libby P, Nathan DM, Abraham K et al (2005) Report of the National Heart, Lung, and Blood Institute-National Institute of Diabetes and Digestive and Kidney Diseases Working Group on Cardiovascular Complications of Type 1 Diabetes Mellitus. Circulation 111:3489-3493

2. Devaraj S, Glaser N, Griffen S, Wang-Polagruto J, Miguelino E, Jialal I (2006) Increased monocytic activity and biomarkers of inflammation in patients with type 1 diabetes. Diabetes 55:774-779

3. Mullick AE, Tobias PS, Curtiss LK (2006) Toll-like receptors and atherosclerosis: key contributors in disease and health? Immunol Res 34:193-209

4. Devaraj S, Dasu MR, Rockwood J et al (2008) Increased toll-like receptor (TLR) 2 and TLR4 expression in monocytes from patients with type 1 diabetes: further evidence of a proinflammatory state. J Clin Endocrinol Metab 93:578-583

5. Wagner H (2006) Endogenous TLR ligands and autoimmunity. Adv Immunol 91:159-173

6. Osterloh A, Breloer M (2008) Heat shock proteins: linking danger and pathogen recognition. Med Microbiol Immunol 197:1-8

7. Park JS, Gamboni-Robertson F, He Q et al (2006) High mobility group box 1 protein interacts with multiple Toll-like receptors. Am J Physiol Cell Physiol 290:C917-C924

8. Nagai K, Arai H, Yanagita M et al (2003) Growth arrest-specific gene 6 is involved in glomerular hypertrophy in the early stage of diabetic nephropathy. J Biol Chem 278:18229-18234

9. Ghanim H, Mohanty P, Deopurkar R et al (2008) Acute modulation of toll-like receptors by insulin. Diabetes Care 31:1827-1831

10. Creely SJ, McTernan PG, Kusminski CM et al (2007) Lipopolysaccharide activates an innate immune system response in human adipose tissue in obesity and type 2 diabetes. Am J Physiol Endocrinol Metab 292:E740-E747 